\title{
Electrical conductivity of oxidized-graphenic nanoplatelets obtained from bamboo: Effect of the oxygen content
}

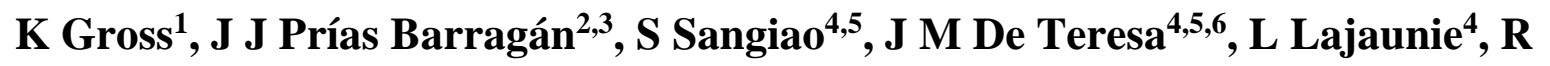 \\ Arenal $^{4,7}, \mathrm{H}$ Ariza Calderón ${ }^{2}$ and P Prieto ${ }^{1}$ \\ ${ }^{1}$ Center of Excellence on Novel Materials, Universidad del Valle, P. O. Box 25157, Cali, Colombia \\ ${ }^{2}$ Interdisciplinary Institute of Sciences, Universidad del Quindı, Carrera 15 Calle 12 Norte, 630001 \\ Armenia, Colombia \\ ${ }^{3}$ Electronic Instrumentation Technology Program, Universidad del Quindı, P. O. Box 661, Armenia, \\ Colombia \\ ${ }^{4}$ Laboratorio de Microscopías Avanzadas (LMA), Instituto de Nanociencia de Aragón (INA), \\ Universidad de Zaragoza, Zaragoza, Spain. \\ ${ }^{5}$ Departamento de Física de la Materia Condensada, Universidad de Zaragoza, 50009 Zaragoza, \\ Spain. \\ ${ }^{6}$ Instituto de Ciencia de Materiales de Aragón (ICMA), CSIC - Universidad de Zaragoza, 50009 \\ Zaragoza, Spain \\ ${ }^{7}$ ARAID Foundation, 50018 Zaragoza, Spain.
}

Email: katherine.gross@correounivalle.edu.co,jiprias@uniquindio.edu.co

\begin{abstract}
Large-scale production of graphene and reduced-graphene oxide (rGO) requires low-cost and ecofriendly synthesis methods. We employed a new, simple, cost-effective pyrolytic method to synthetize oxidized-graphenic nanoplatelets (OGNP) using bamboo pyroligneous acid (BPA) as source. Thorough analyses via high-resolution transmission electron microscopy and electron energy-loss spectroscopy provides a complete structural and chemical description at the local scale of these samples. In particular, we found that at the highest carbonization temperature the OGNP-BPA are mainly in $\mathrm{sp}^{2}$ bonding configuration ( $\mathrm{sp}^{2}$ fraction of $87 \%$ ). To determine the electrical properties of single nanoplatelets, these were contacted by Pt nanowires deposited through focused-ion-beam-induced deposition techniques. Increased conductivity by two orders of magnitude is observed as oxygen content decreases from 17 to $5 \%$, reaching a value of $2.3 \times 10^{3} \mathrm{~S} / \mathrm{m}$ at the lowest oxygen content. Temperature-dependent conductivity reveals a semiconductor transport behavior, described by the Mott three-dimensional variable range hopping mechanism. From the localization length, we estimate a band-gap value of $0.2(2) \mathrm{eV}$ for an oxygen content of $5 \%$. This investigation demonstrates the great potential of the OGNP-BPA for technological applications, given that their structural and electrical behavior is similar to the highlyreduced rGO sheets obtained by more sophisticated conventional synthesis methods.
\end{abstract}

\section{Introduction}


Graphene oxide (GO) and reduced GO (rGO) have attracted research attention because of their interesting tunable properties, as well as their use as precursors for mass production of graphene sheets [1-9]. Tunability, in combination with the superior properties intrinsic of graphene, makes rGO promising candidate for next-generation nanoelectronic devices. Graphene oxide can be described as an oxidized form of graphene, decorated mostly by hydroxyl and epoxy functional groups distributed randomly along the hexagonal network of carbon atoms and, additionally, by carboxyl groups positioned at the sheet edges [10-14]. Nevertheless, for the case of oxygen content below $25 \%$, it has been predicted that only hydroxyl species can be present [12]. By nature, GO is electrically insulating and, thus, cannot be used without further processing as a conductive nanomaterial. However, it has been shown that reduction of GO is an effective method to obtain metallic or semiconductor structures [4, 15-18]. Reduction of GO is typically carried out through chemical reduction [3, 5, 19], thermal annealing [14, 18, 20], or a combination of both [21]. However, a considerable number of structural defects are concomitantly created during these processes having strong implications on the nature of electrical transport in these materials. Transition from insulator to conducting state via chemical/thermal reduction to derive pure graphene from GO for large-scale production is still not a trivial issue. Long reaction times, multiple steps, and toxicity of the chemical agents are some barriers that still need to be solved.

It is known that C-O bonds present in GO modify the atomic structure of pristine graphene. A comprehensive analysis of the atomic structure of GO and rGO is considered an important step to understand the electronic transport of this material. First principles calculations [22] have shown that by changing the oxidation level and the relative compositions of the epoxy and hydroxyl groups, moderate bandgaps can be opened. Several theoretical models of atomic structure have been also proposed in the literature, such as the Hoffmann model [23], Lerf-Klinowski model [24], Scholz-Boehm model [25], Nakajima-Matsuo model [26], Ruess model [27], Zsabo-Dekany model [28]. On the other hand, experimental high-resolution transmission electron microscopy (HRTEM) investigations have helped to elucidate the distribution of the oxygen-functional groups as well as the kind of structural- and topological defects [29-33]. Nevertheless, until now the exact atomic structure of GO and rGO is still unresolved. In general, recent reports describe GO sheets as a random distribution of oxidized regions bearing the oxygenated functional groups, coexisting with non-oxidized regions wherein most of the carbon atoms preserve $\mathrm{sp}^{2}$ hybridization.

Electrical conductivity and oxygen concentration are strongly correlated in rGO. In general, it has been shown that by increasing the reduction level of rGO, i.e., by restoring $\mathrm{sp}^{2}$ hybridization, room temperature conductivity tends to increase $[15,17,18,34]$. In rGO, electrical conduction is caused by $\mathrm{sp}^{2}$ hybridization due to the presence of delocalized electrons ( $\pi$ orbital), which freely move throughout the graphitic structure. In oxygenized areas containing a high amount of carbon domains showing $\mathrm{sp}^{3}$ hybridization, this material behaves as insulator. Charge transport mechanism in a landscape of coexisting $\mathrm{sp}^{2} / \mathrm{sp}^{3}$ regions is akin to that of disordered semiconductors where electron localization and hopping conduction play a significant role. In fact, depending on oxygen content, rGO-based materials 
undergo insulator-semiconductor-semimetal transitions with reduction. Studies varying the $\mathrm{sp}^{2}$ fraction have shown that diverse types of conduction mechanisms can take place depending on whether the rGO sheet is lightly or highly reduced [43, 47]. Specifically, Eda et. al., [43] report two different mechanisms describing the conductivity for low-range and high-range $\mathrm{sp}^{2}$-fraction. While the former is dominated by hopping or tunneling amongst the $\mathrm{sp}^{2}$ clusters, the latter can be described by percolation process. For electrically conductive systems, i.e. highly reduced rGO, graphene-based polymer nanocomposites, percolation theory has been developed to describe the geometrical and temperature dependences of the conductivity [Mutlay and Tudoran]. On the other hand, for systems showing semiconductor behavior [12, 22, 35], Amirhasan Nourbakhsh et al., [36] report bandgap generation as a result of oxygen functionalization of graphene for $\mathrm{O} / \mathrm{C}$ ratio higher than $11.1 \%$. However, authors in [37] claimed that rGO is expected to have a metallic-like behavior for O/C ratio up to $25 \%$. Until now, consensus has not been reached about the $\mathrm{sp}^{2}$ fraction at which the transport mechanism changes from metallic- to semiconductor-like behavior; that is, why an experimental study in a range of low oxygen content, lower than $25 \%$, is of great relevance to gain further insight in the electrical behavior in this low-oxygen regime.

Regarding the temperature dependence of the conductivity in rGO exhibiting semiconductorlike transport behavior, different conduction mechanisms have been reported [3, 4, 16, 38-42]. Authors in [43] found that while at a higher temperature regime of well-reduced GO thermally-excited carriers dominate the conduction, the low-temperature regime must be described through a variable-range hopping (VRH) model. The temperature-dependent longitudinal conductivity considering the VRH model can be described by the general form: $\ln \sigma(\mathrm{T}) \alpha\left(\mathrm{T}_{0} / \mathrm{T}\right)^{1 / \mathrm{d}+1}$, where $d$ describes the dimensionality of the system [44]. Thus, $d=2$ and 3 correspond to the Mott VRH conduction mechanism, which was proposed for 2D and 3D systems, respectively, without considering Coulomb interaction. When Coulomb interaction between initial and final hopping sites exists, Efros and Shklovskii [45] proposed the case of $d=1$ for the long-range hole-electron interaction at the Fermi level. Finally, $d=0$ corresponds to the Arrhenius-type for thermal-activated conduction mechanism. It is worth mentioning that the first three possibilities have also been applied for analysis of charge transport mechanisms in non-crystalline materials [46].

Large-scale graphene production obtained via rGO exfoliation is still difficult. Defect formation or intercalated species like water molecules make the complete control of exfoliation a challenge [48]. In general, to avoid sophisticated GO reduction processes and eventual environmental contamination from the reducing agents, simple, green, and cost-effective methods are highly desirable. In a recent work, we reported on a new, simple, cost-effective method for the synthesis of oxidized-graphenic platelets by using bamboo pyroligneous acid (BPA) as source, showing similar vibrational properties as rGO obtained via conventional chemical methods [49]. The present work seeks to gain insight into the local atomic structure and electrical conductivity behavior of OGNP-BPA, given that research on these kinds of properties is of relevance for evaluating the impact on technological applications of this carbon 
material obtained through the new fabrication method using natural bamboo as source. We report the electrical transport characterization of single OGNP-BPA for oxygen content ranging from 5 to $17 \%$. For electrical characterization, single nanoplatelets were contacted by focused-ion-beam-induced deposition of Pt nanowires, allowing the intrinsic resistance to dominate over the contact resistances. Measurements of current-voltage characteristic at room- $\mathrm{T}$ and temperature dependent resistivity demonstrate that OGNP-BPA show comparable conductivity values as the conventionally synthetized rGO sheets (referenced above) with values around $2.3 \times 10^{3} \mathrm{~S} / \mathrm{m}$ at room temperature and semiconductor-like behavior with bandgap value up to $0.30 \mathrm{eV}$ for the highest oxygen concentration.

\section{Experimental}

Oxidized-graphenic nanoplatelets studied in this work were obtained by thermal decomposition method using bamboo pyroligneous acid (BPA) extracted from Guadua angustifolia Kunth, macana biotype. BPA was obtained by a first pyrolysis process at $973 \mathrm{~K}$ using a pyrolysis system under a controlled nitrogen atmosphere. We, thereafter, used the BPA as precursor for OGNP. For this, a second pyrolysis process using the BPA was carried out at different carbonization temperatures $\left(T_{C A}\right)$, with $T_{C A}$ varying from 673 to $973 \mathrm{~K}$. Varying $T_{C A}$ allows modifying the crystal structure and oxygen-containing functional groups present in the samples. When the second carbonization process is completed, oxidized-graphenic material is obtained as macro foam, which then turns into nanoplatelets powders by mechanical grinding with a ceramic hand mortar. A detailed description of the method for obtaining oxidized-graphenic material can be found in a previous work [49].

HRTEM, electron diffraction (ED) and electron energy-loss spectroscopy (EELS) spectra were performed on a FEI Titan Cube microscope operating at $80 \mathrm{kV}$ and equipped with a Cs image corrector and a Gatan Tridiem spectrometer. The EELS experiments were performed at liquid nitrogen temperature to hinder carbon contamination. The probe diameter for EELS acquisition was around 30 $\mathrm{nm}$. Convergence and collection angles were 4.6 and $20 \mathrm{mrad}$, respectively. The energy resolution, measured as the full width at half maximum of the zero loss peak (ZLP), was $0.8 \mathrm{eV}$ with an energy dispersion of $0.2 \mathrm{eV} /$ pixel and $0.6 \mathrm{eV}$ for a dispersion of $0.05 \mathrm{eV} /$ pixel. The first dispersion was used for elemental quantification and the second one for fine-structure analysis and to determine the $\mathrm{sp}^{2}$ fraction. The typical acquisition times for core-loss and low-loss spectra were 16 and 0.2 seconds, respectively. Background subtraction for the core-loss spectra was performed by modeling the usual inverse power law function and multiple scattering was then removed by Fourier-ratio deconvolution [50] with the low-loss spectrum obtained for exactly the same region of the sample. Oxygen content was then determined from EELS elemental quantification. The $\mathrm{sp}^{2}$ ratio was determined by using spectra of a highly ordered pyrolytic graphite sample obtained under the same experimental conditions. The exact procedure is explained elsewhere [51].

Mass density and absolute thickness of the analyzed nanoplatelets were obtained by analyzing the low-loss spectra. For this purpose, the low-loss spectra were deconvoluted by the ZLP by using the 
PEELS program [52] and the single-scattering spectra were obtained by following Stephen's procedure [53], which also provided the $t / \lambda$ ratio ( $t$ is the thickness of the analyzed crystal and $\lambda$ is the inelastic mean free path). For each spectrum, the $(\pi+\sigma)$ volume plasmon was then modelled by the Drude model over the top $75 \%$ of the plasmon peak intensity (Figure S1 in Supplementary Information) in order to extract the plasmon energy, $E_{P}$. This value was then used in the following relation [54] to determine the mass density and by assuming that carbon contributes to four valence electrons and oxygen contributes to six:

$$
\rho=\frac{E_{P}^{2} \times m^{*} \times \epsilon_{0} \times \mu \times\left(12+4 X_{O}\right)}{\hbar^{2} \times e^{2} \times\left(4+2 X_{O}\right)}
$$

Where $\rho$ is the mass density, $E_{P}$ is the plasmon energy, $\varepsilon_{0}$ is the vacuum permittivity, $\mu$ is the atomic mass unit, $e$ is the elementary charge, $\hbar$ is the Dirac constant, $X_{O}$ is the atomic fraction determined from core-loss EELS spectra (and by assuming that $X_{C}+X_{O}=1$ ), and $m^{*}$ is the effective electron band mass taken as $m^{*}=0.87 m[55,56]$. Sample thickness was determined by estimating $\lambda$ with the modified Iakoubovskii formula [54, 57], using mass density and oxygen fraction as inputs and yielding a value of $\lambda=106 \mathrm{~nm}$. This approach has been shown to yield a reasonable estimation of absolute thickness [58].

We also used energy dispersive X-ray spectroscopy (EDS) in a scanning electron microscope (SEM, see below) to complete the compositional study and check oxygen content (atomic percent, $C_{O}$ ). In order to perform electrical measurements of individual nanoplatelets, the samples were prepared by suspending OGNP-BP in isopropanol and depositing them in proximity to the metallic contact pads (fabricated firstly by optical lithography) on a $\mathrm{SiO}_{2} / \mathrm{Si}$ substrate. Individual nanoplatelets were then located at low voltages $(<5 \mathrm{kV}$ ) using a SEM equipped with a focused ion beam (FIB) (Helios 650 model by FEI). The focused ion beam induced deposition (FIBID) technique was used to deposit four Pt lead wires to connect the nanoplatelets to the metallic contact pads where micro-probes were positioned. The Pt-based contacts deposited optimally by FIBID allow low-contact resistivity to perform noise-free electrical characterization of micro- or nanostructures [59, 60]; for more details of this technique please refer to [61]. The SEM images of the experimental setup displaying the micro-probes, the metallic contact pads, and Pt wires on a single OGNP-BPA are shown in figure 1. The electrical measurements were performed by injecting current in a range of $\pm 6 \mu \mathrm{A}$ on the two external contacts using a Keithley 6220 DC current source; while the voltage was measured at the two internal contacts with a Keithley 2182A nanovoltmeter (see the bottom right panel in figure 1). Temperature-dependent measurements of the resistance were performed within a vacuum chamber using a closed-cycle Helium cryostat system.

\section{Results and Discussion}

\subsection{Morphology, structural and chemical investigations}


To gain further insight on the structural and chemical properties, HRTEM and EELS analyses were performed on the nanoplatelets. A combination of TEM and EELS is a powerful tool to get access to valuable information with nanometer or sub-Ångström resolution [62-65]. In particular, a wealth of
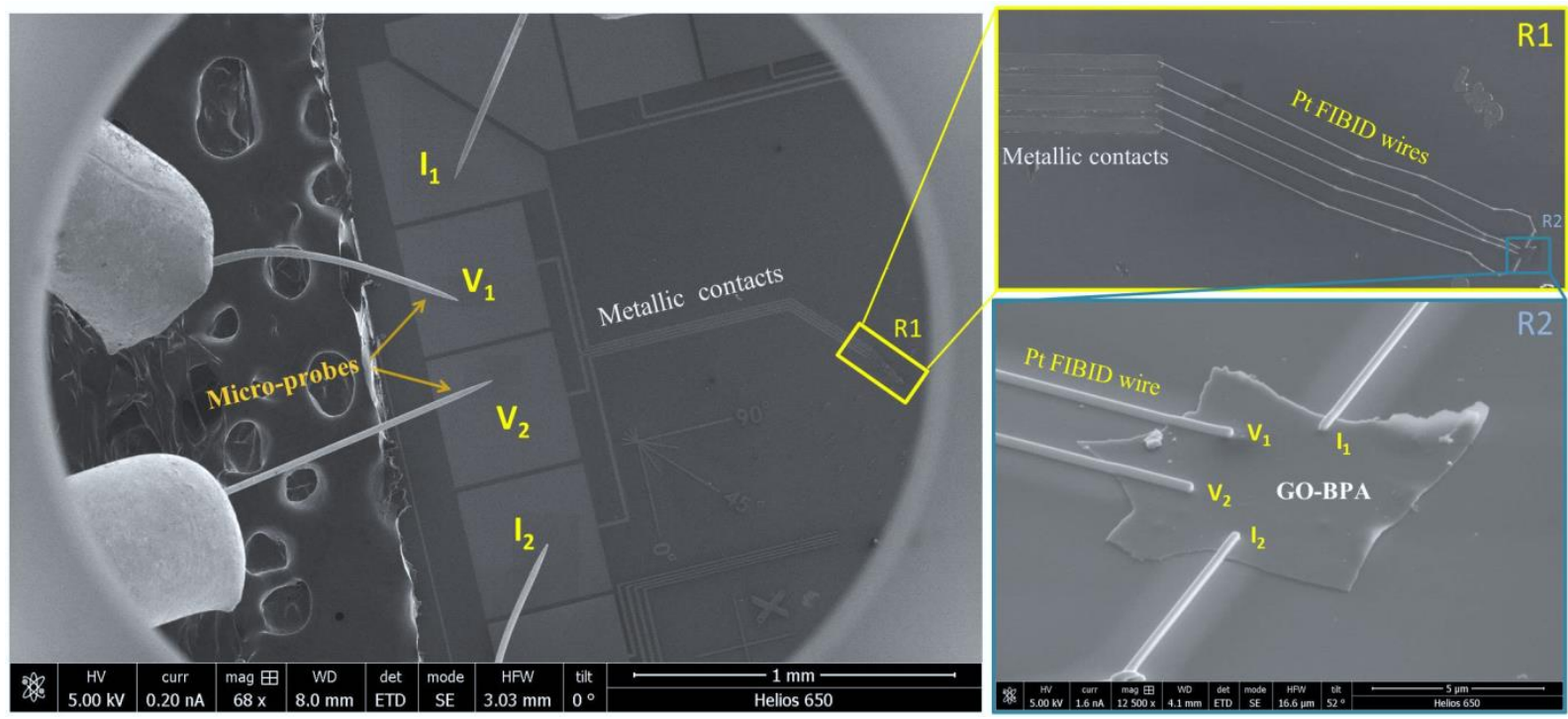

Figure 1. Scanning electron microscopy image of the experimental setup displaying the micro-probes and the metallic contact pads (left), zoomed area of region R1 showing the Pt-wires grown via FIBID technique on a $\mathrm{SiO}_{2} / \mathrm{Si}$ substrate (top right), and zoomed area of region R2 showing a typical OGNPBPA with lateral dimensions around $7 \mu \mathrm{m}$ and thickness $\mathrm{t}<100 \mathrm{~nm}$, and Pt contacts to perform the electrical measurements (bottom right).

chemical information related to elemental quantification, mass density, local chemical environment, valence state and bonding hybridization can be extracted from carbon nanostructures and other materials [55, 66, 67]. Figures 2(a) and 2(b) show TEM micrographs of the samples prepared with the highest $T_{C A}(973 \mathrm{~K})$ and the lowest one $(673 \mathrm{~K})$, respectively. Thin nanoplatelets are clearly visible upon the TEM carbon membrane. Nanoplatelets size is typically in the order of a dozen micrometers and presents a thickness, as estimated from low-loss EELS spectra, ranging between 25 and $60 \mathrm{~nm}$.

Figures 2(c) and 2(d) show two HRTEM micrographs of the samples prepared with a $T_{C A}$ of 973 and $673 \mathrm{~K}$, respectively. The presence of disordered graphitic clusters is clearly highlighted (blue arrows in figure 2(c)) for the sample prepared at $T_{C A}$ of $973 \mathrm{~K}$, while the micrograph of the sample prepared at $T_{C A}$ of $673 \mathrm{~K}$ is more characteristic of disordered materials. The selected area electron diffraction (insets of figures 2(a) and 2(b)) show diffuse rings characteristic of disordered and low-order material. The random attachments of the oxygen functional groups on the top and bottom surfaces of OGNP-BPA monolayers play a crucial role in determining the stacking order; because they: $i$ ) break the symmetry of the subjacent honeycomb carbon lattice; ii) introduce a slight roughness, which is originated from out-of-plane lattice distortions of the C-O bonds and iii) increase the interlayer spacing (XRD diffractograms in Figure S2 in supplementary information, confirm an increased interlayer spacing with increasing oxygen content). Due to the higher interlayer spacing, individual layers show rotational -and tilt- disorder relative to their neighbors and thus, no preferred stacking orientation. Nevertheless, it is 
worth to mention that diffracted rings are better defined for the sample prepared with a TCA of $973 \mathrm{~K}$ and, thus, confirm the higher local order of this sample.

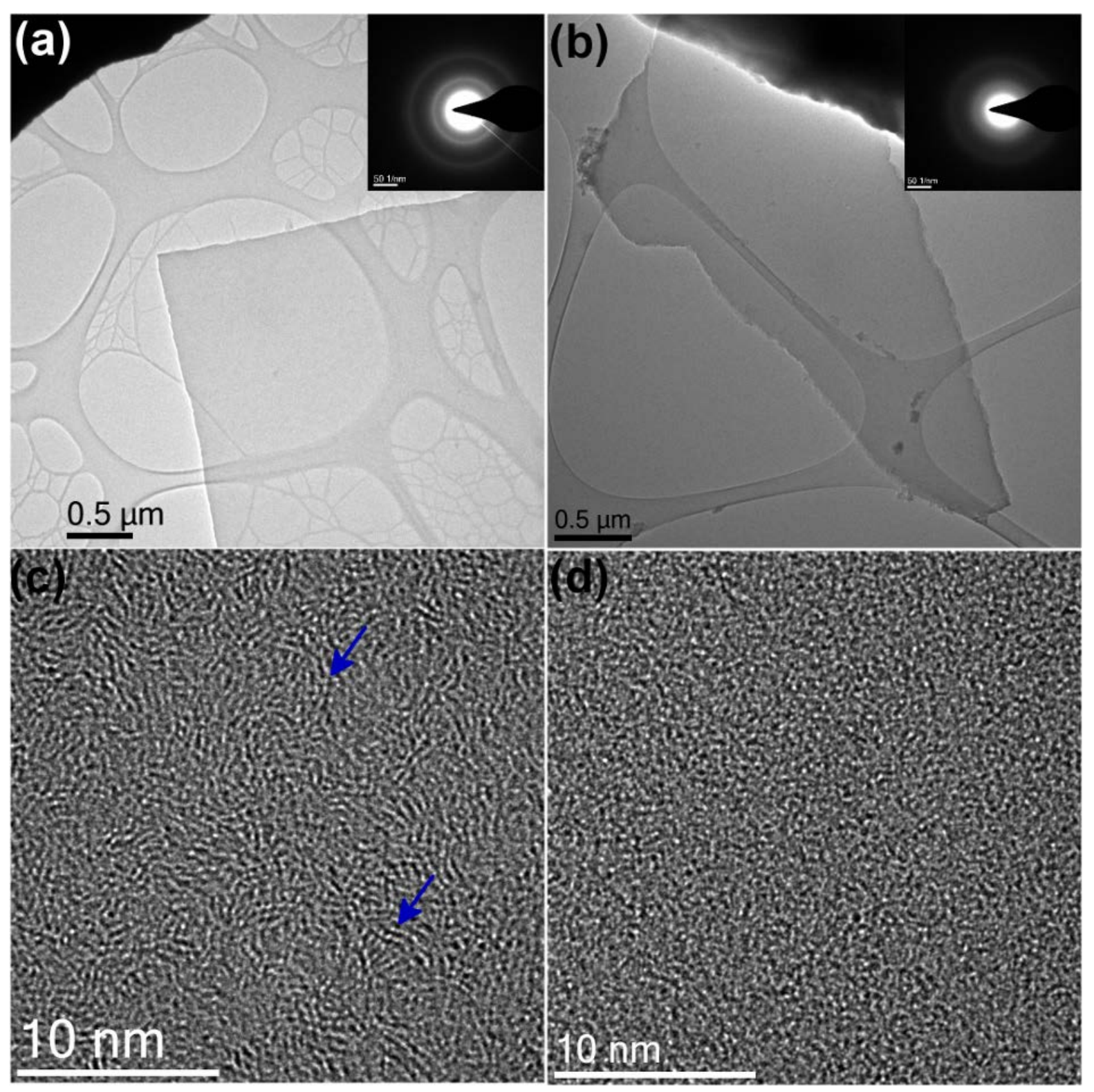

Figure 2. (a) and (b) Low-magnification TEM micrographs of the sample prepared with a $T_{C A}$ of 973 and $673 \mathrm{~K}$, respectively. Insets show the selected area electron diffraction. (c) and (d) HRTEM micrographs of the sample prepared with a $T_{C A}$ of 973 and $673 \mathrm{~K}$, respectively. The blue arrows highlight the disordered graphitic clusters.

Despite the short range crystal order and the incoherence along the c-direction due the high interlayer spacing values, accurate EELS measurements gave evidence about the high level of sp2bonds in our nanoplatelets. Figure 3(a) shows the EELS spectra of the samples prepared at 973 and $673 \mathrm{~K}$. The C-K edge (roughly situated between 280 and $325 \mathrm{eV}$ ) and the O-K edge (situated around $530 \mathrm{eV}$ ) are clearly visible. In addition, a change of the slope around $400 \mathrm{eV}$ (highlighted by a red 
arrow in figure 3(a)) indicates the presence of nitrogen at chemical trace level. The XPS analyses performed on the whole powders confirmed the presence of nitrogen with a concentration below $1 \%$ for all the samples. The $\mathrm{O} /(\mathrm{C}+\mathrm{O})$ ratio determined by EELS is around $3 \%$ and $12 \%$ for the samples prepared at 973 and $673 \mathrm{~K}$, respectively, although it is important to bear in mind that precise and accurate EELS quantification requires comparison with reference materials [67]. Figure 3(b) shows the C-K edge of the two samples with a higher resolution. Between 290 and $305 \mathrm{eV}$, the spectrum of the

sample prepared at $973 \mathrm{~K}$ presents characteristic graphite-like fine structures (blue arrows in figure 3(b)), indicating the influence of band-structure effect. This is of importance because it confirms the presence of a short-range crystalline order and shows that locally the carbon is mainly in threefold $\left(\mathrm{sp}^{2}\right)$ bonding configuration. In the same energy range, the C-K edge of the sample prepared at $673 \mathrm{~K}$ is featureless and similar to the spectra of amorphous carbons [51, 68]. At $286.7 \mathrm{eV}$, the spectrum of the sample prepared at $673 \mathrm{~K}$ show a supplementary peak (green arrow in figure 3(b)), which could be linked to the presence of oxygen. For instance, $\mathrm{C}=\mathrm{O}$ bonds give rise to transitions to $\pi^{*}$ states in the energy range between 287.7 and $288.3 \mathrm{eV}$ and $\mathrm{C}-\mathrm{H}$ bonds give rise to transitions to $\sigma^{*}$ states in the energy range between 287.6 and $288.2 \mathrm{eV}$ [69, 70]. However, the determination of the origin of this supplementary transition based solely on the energy position is particularly difficult. From the core-loss spectra, the $\mathrm{sp}^{2}$ fraction ( $\mathrm{sp}^{2}$ character/ $\left(\mathrm{sp}^{2}+\mathrm{sp}^{3}\right.$ characters)) has been derived and is equal to $87 \%$ and $66 \%$ for the samples prepared at 973 and $673 \mathrm{~K}$, respectively. The decreased $\mathrm{sp}^{2}$ character is also confirmed by the low-loss spectra, which show a strong decrease of the $\pi$ plasmon for the same sample (Figure S1 in Supplementary Information). In addition, the mass density of the nanoplatelets was calculated from the low-loss spectra and is around 1.80 and $1.92 \mathrm{~g} \mathrm{~cm}^{-3}$ for the samples prepared with a $T_{C A}$ of 973 and $673 \mathrm{~K}$, respectively. These values constitute an intermediate between the values corresponding to glassy carbon and pure graphite (1.44 and 2.27 g.cm ${ }^{-3}$, respectively) [71].The results obtained via electron diffraction, HRTEM, and EELS analyses are consistent and provide a complete structural and chemical description at the local scale of these samples. During synthesis, the lowest $T_{C A}$ yields nanoplatelets that are mostly disordered and with an $\mathrm{O} /(\mathrm{C}+\mathrm{O})$ ratio of $12 \%$. On the other hand, the highest $T_{C A}$ yields nanoplatelets that show mostly $\mathrm{sp}^{2}$ bonding configuration with a short-range crystalline order and an $\mathrm{O} /(\mathrm{C}+\mathrm{O})$ ratio of $3 \%$. These important chemical and structural modifications should have a strong impact on the electrical properties. 

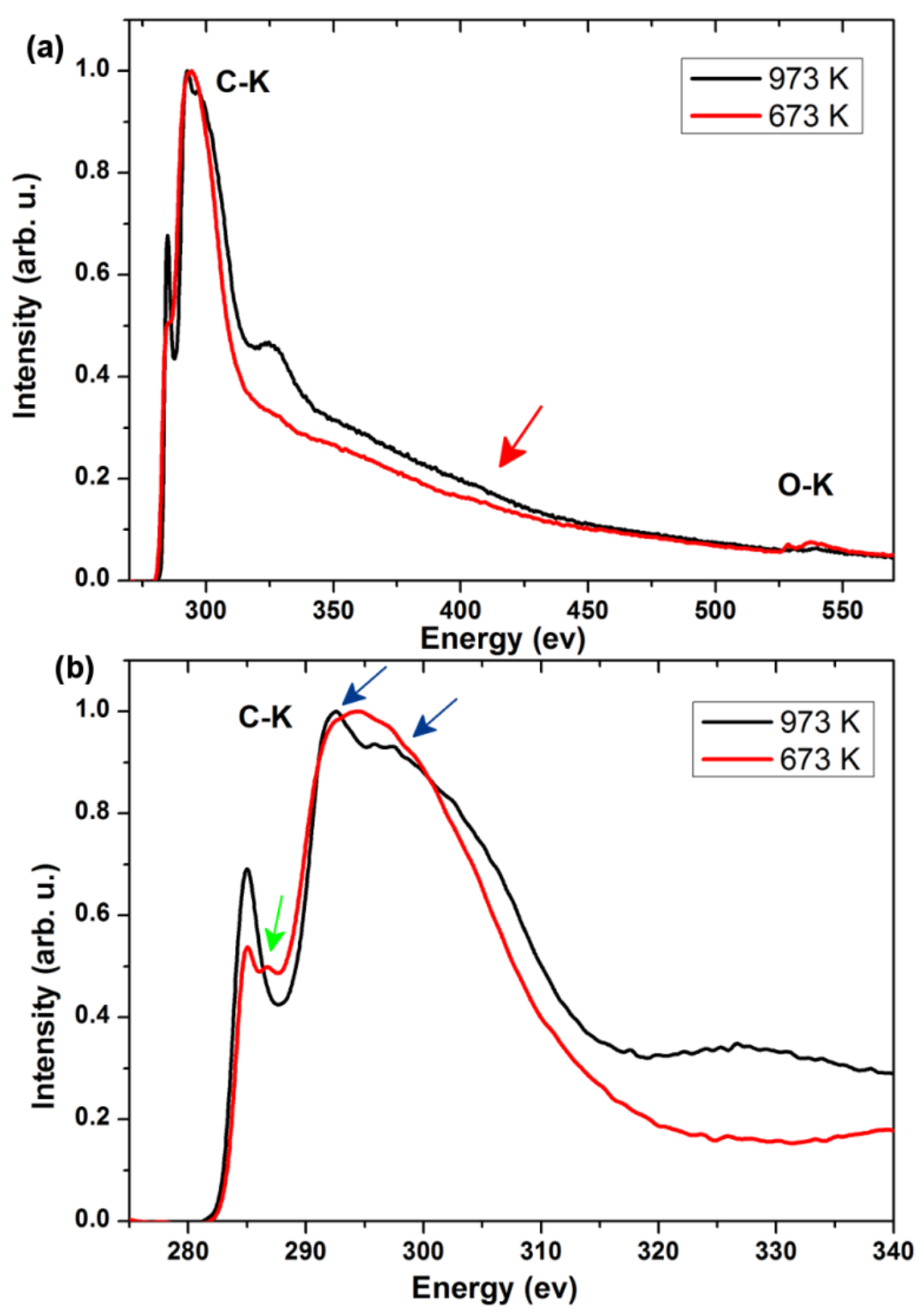

Figure 3. (a) EEL spectra of the samples prepared with a $T_{C A}$ of $973 \mathrm{~K}$ (black curve) and $673 \mathrm{~K}$ (red curve) and recorded with a dispersion of $0.2 \mathrm{eV} /$ pixel. The red arrow highlights the position of the $\mathrm{N}$ K edge. (b) EEL spectra of the C-K edge recorded with a dispersion of $0.05 \mathrm{eV} /$ pixel. The blue arrows highlight the graphitic fine structures of the sample prepared at $973 \mathrm{~K}$ and the green arrow highlights the supplementary peak observed at $286.7 \mathrm{eV}$ for the sample prepared at $673 \mathrm{~K}$ (see text for more details). All the spectra have been submitted as references to the EELS database [72].

\subsection{Electrical characterization}

\subsubsection{Room temperature conductivity: Effect of the oxygen content}

Variation of synthesis conditions, specifically carbonization temperature, leads to modification of the oxygen-containing functional groups, as well as of the crystal structure in OGNP-BPA, as presented above and also confirmed by X-Ray diffraction (Figure S2 in Supplementary Information) and by other techniques [49]. For electrical characterization we focused on OGNP-BPA obtained at higher $T_{C A}$ (873 
and $973 \mathrm{~K}$ ), which ensures an improved crystal structure, as confirmed by HRTEM (Figure S3 in Supplementary Information). Under these temperature conditions, the oxygen content is below 20\%, which is our interest, given that it is known that within this oxygen regime the electrical behavior in GO is still unclear. Room temperature electrical conductivity was obtained via current-voltage $(I-V)$ measurements for the oxygen atomic concentrations $C_{O}=5,8,13$ and $17 \%$. All $I-V$ curves exhibit ohmic behavior along the entire current range $( \pm 6 \mu \mathrm{A})$, allowing calculation of conductivity values from the slope and geometric parameters of the nanoplatelets obtained via SEM. Room temperature conductivity as a function of $C_{O}$ is shown in figure 4. Decreased oxygen content from 17 to $5 \%$ resulted in conductivity rise by two orders of magnitude, starting from $6.4 \times 10^{1} \mathrm{~S} / \mathrm{m}$ and reaching a value of $2.3 \times 10^{3}$ $\mathrm{S} / \mathrm{m}$ at the lowest oxidation degree. These values are comparable with literature reports on reduced GO obtained through chemical/thermal reduction methods, where it has been shown that conductivity can vary from 5 to $5 \times 10^{4} \mathrm{~S} / \mathrm{m}$, depending on the degree of reduction [2, 47]. When $\mathrm{O}$ atoms from the functional groups are released, the graphene oxide lattice, rich in $\mathrm{sp}^{3}$ hybridization, transforms to a graphene-like structure due to the restoration of $\mathrm{sp}^{2}$ hybridization and, thereby, its conductivity increases. Also, rGO sheets, obtained by chemical reduction, exhibit only moderate room-temperature conductivities, predominantly as a consequence of crystal defects (like extended holes) that cannot be healed during the reduction process. Formation of holes in our OGNP-BPA can be considered minimal, as evidenced above by HRTEM examinations.

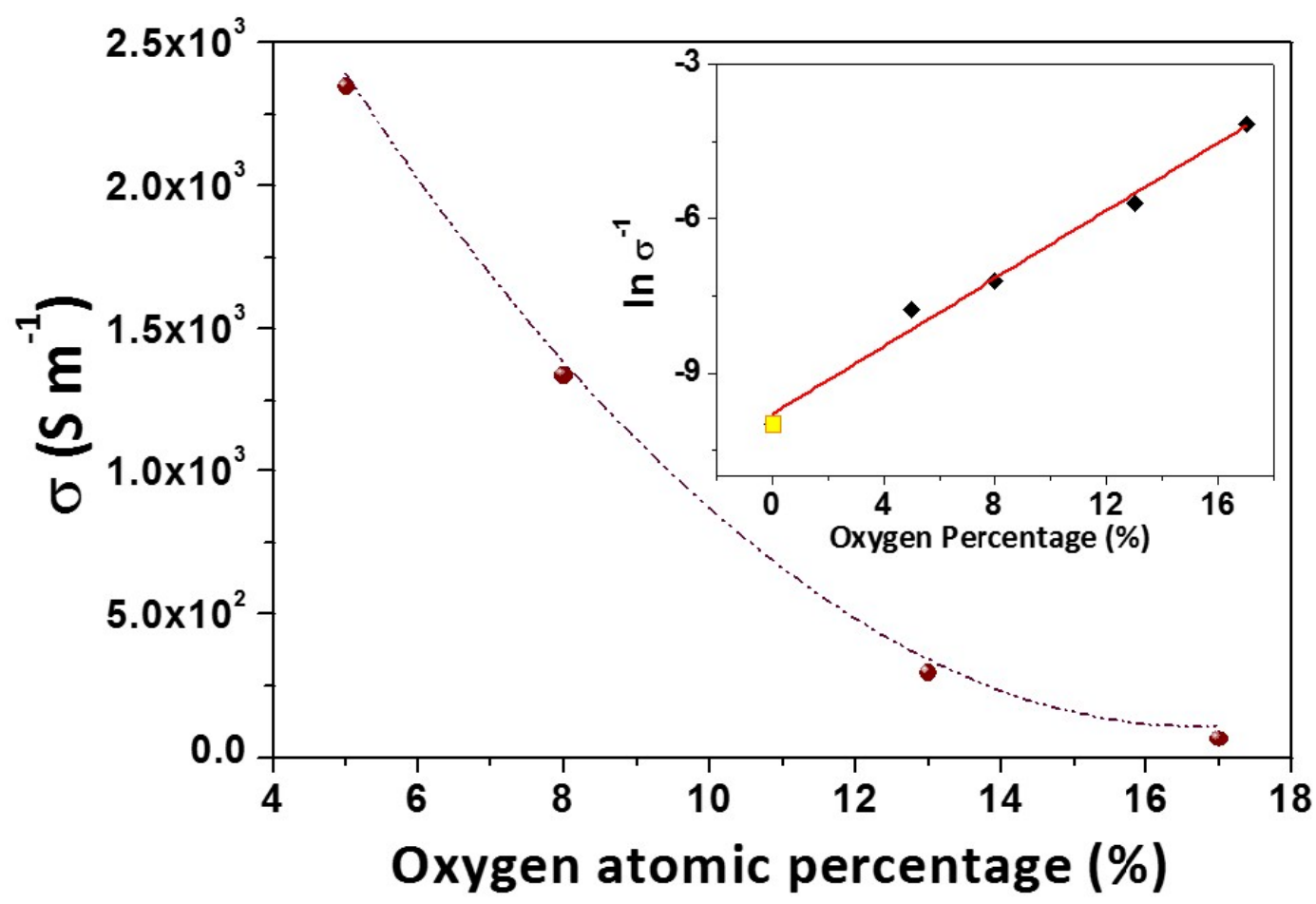

Figure 4. Electrical conductivity as a function of oxygen content $\left(C_{O}=5,8,13\right.$, and $\left.17 \%\right)$. The line is a guide to the eye. Inset: Fit (solid line) to the experimental data (full diamonds) using the expression for carrier concentration for an intrinsic semiconductor. 
To gain more insight on the dependence of conductivity on oxygen concentration, shown in figure 4, we consider the relation $\eta \propto \exp \left(-\frac{E_{g}}{2 k_{B} T}\right)$, which relates the carrier concentration to the temperature and bandgap energy, $E_{g}$, for an intrinsic semiconductor; with $k_{B}$ being the Boltzmann constant. Thus, if a linear dependence of the bandgap on oxygen concentration is assumed, as proposed in [12], and because $\sigma$ is proportional to $\eta, \ln (\sigma)$ will behave linearly with $C_{O}$. Our experimental data, inset in figure 4, agree well with these theoretical assumptions. For contextualization, we compare our data in this plot with that reported for graphite (yellow square). As can be seen, the conductivity for the lowest value of oxygen content found in OGNP-BPA is still around one order of magnitude lower than the value for graphite $\left(2 \times 10^{4} \mathrm{Sm}^{-1}\right)$, thereby, elucidating the direct effect of the oxygen functionalized groups on electrical conductivity.

By using the explicit relationship $\sigma=\sigma_{0} \exp \left(-\mathrm{E}_{\mathrm{g}} /\left(2 \mathrm{k}_{\mathrm{B}} \mathrm{T}\right)\right)$ with $\sigma_{0}=2 \times 10^{4} \mathrm{Sm}^{-1}$, as reported for graphite at $T=300 \mathrm{~K}$, and considering conductivity values for $C_{O}=5,8,13$, and $17 \%$, reported in figure 4 , we estimate the energy bandgap values as a function of $C_{O}$, as shown in figure 5 , along with a fit line using the general quadratic dependence of the bandgap with the scatter center $X$ applied for semiconductors [73, 74]. Bandgap energy shows a variation from 0.30 to $0.11 \mathrm{eV}$ by decreasing the oxygen content. Agreement between our experimental results and theoretical prediction is quite remarkable, providing evidence of oxygen-mediated charge-transport scattering in our OGNP-BPA and suggesting that the OGNP-BPA exhibit narrow-gap semiconductor behavior.

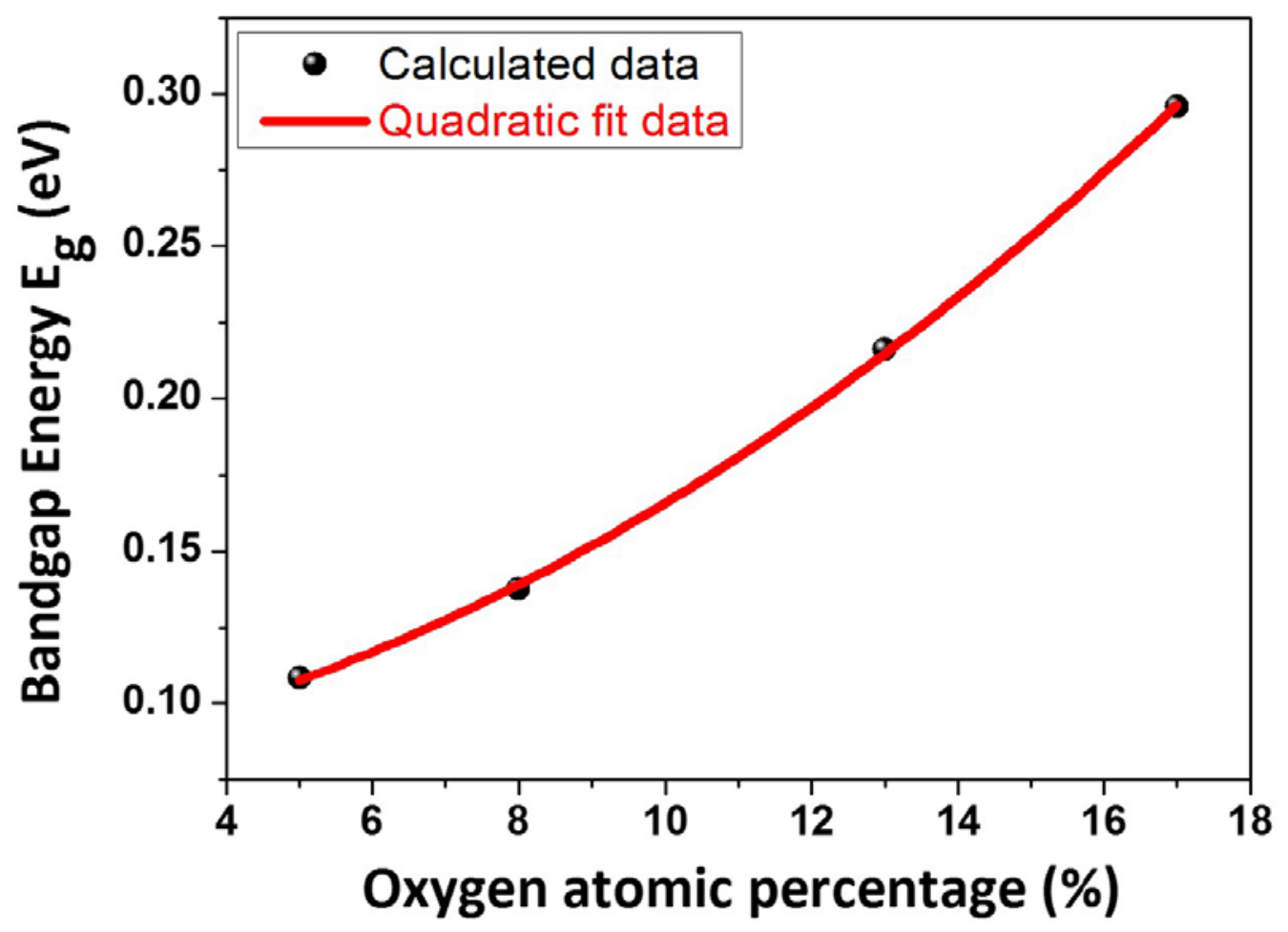

Figure 5. Bandgap $\left(E_{g}\right)$ of OGNP-BPA plotted vs. their corresponding oxygen atomic percentage; red solid line is the fit using the general quadratic dependence of the bandgap with the scatter center $\mathrm{X}$ applied for semiconductors [73, 74]. 


\subsubsection{Temperature dependence of electrical conductivity}

It has been reported that temperature dependent conductivity in case of semiconductor-like behavior of rGO can be explained by variable-range hoping (VRH) through the localized states [16,?,?]. While in case of electrical conductive system, percolation models are able to fit experimental data [Mutlay and Tudoran]. To gain insight into the energies associated with the charge transport in our OGNP-BPA, the temperature dependence on conductivity is investigated. Figure 6(a) shows the temperature dependence of the resistivity for OGNP-BPA with $C_{O}=5 \%$. As it can be seen, resistivity increases with decreasing temperature exhibiting a semiconductor-like behavior. The total variation of the resistivity in this range is less than one order of magnitude, showing weak temperature dependence - as reported for highly reduced $\mathrm{rGO}$ [47]. We have plotted $\ln \left(\sigma \mathrm{T}^{1 / 2}\right)$ as a function of $\mathrm{T}^{-1 / 4}$ within $25-300 \mathrm{~K}$, which is shown in figure 6(b) along with a best fit line from the VRH model. These results suggest that the charge transport in OGNP-BPA is dominated by the Mott three-dimensional VRH transport behavior. For this case, the temperature dependence on conductivity can be expressed as:

$$
\sigma=\frac{\sigma_{0}}{T^{1 / 2}} \exp \left[-\left(\frac{T_{0}}{T}\right)^{1 / 4}\right]
$$

where $\sigma_{0}$ and $T_{0}$ are given by:

$$
\begin{gathered}
\sigma_{0}=\frac{3 e^{2} v_{p h}}{(8 \pi)^{1 / 2}} \times\left[-\left(\frac{N\left(E_{F}\right)}{\alpha k_{B}}\right)\right]^{1 / 2} \\
T_{0}=\frac{16 \alpha^{3}}{k_{B} N\left(E_{F}\right)}
\end{gathered}
$$

where $v_{p h}$ is the phonon frequency $\left(\approx 10^{13} \mathrm{~Hz}\right)$ at Debye's temperature. $N\left(E_{F}\right)$ is the density of the localized electron states at Fermi's level and $\alpha$ is the inverse localization length of wave function associated with the localized state. As seen from the graph, $\ln \left(\sigma \mathrm{T}^{1 / 2}\right)$ versus $\mathrm{T}^{-1 / 4}$ in figure $6(\mathrm{~b})$, the plot exhibits good linear behavior along the entire $T$ range, having a linearity factor of 0.997 . From the slope and intercept values, parameters $T_{0}=7.1 \times 10^{4} \mathrm{~K}$ and $\sigma_{0}=8.8 \times 10^{3} \mathrm{Sm}^{-1}$ are obtained. Using the extracted values for $T_{0}$ and $\sigma_{0}$, we calculated the density of the localized states at Fermi's Level $N\left(E_{\mathrm{F}}\right)=1.2 \times 10^{21}$ $\mathrm{eV}^{-1} \mathrm{~cm}^{-3}$ and a value for $\alpha$ of $7.78 \times 10^{6} \mathrm{~cm}^{-1}$ according to eq. (2) and (3). Likewise, localization length $\xi=1 / \alpha$ is determined as $1.3 \mathrm{~nm}$. Thus, if assuming a wave function confined inside the graphitic domains of size $\mathrm{D} \sim \xi$ and considering the linear dispersion relation for graphene as $\mathrm{E}(\mathrm{k})=\hbar v_{F} \mathrm{k}$, with $v_{F}$ being the graphene Fermi velocity, and with $\mathrm{k} \sim 1 / 2 \xi$, the bandgap energy can be estimated as $0.22 \pm 0.02 \mathrm{eV}$, in good agreement with the value of $0.25 \mathrm{eV}$ of oxidized graphene reported elsewhere [75]. 

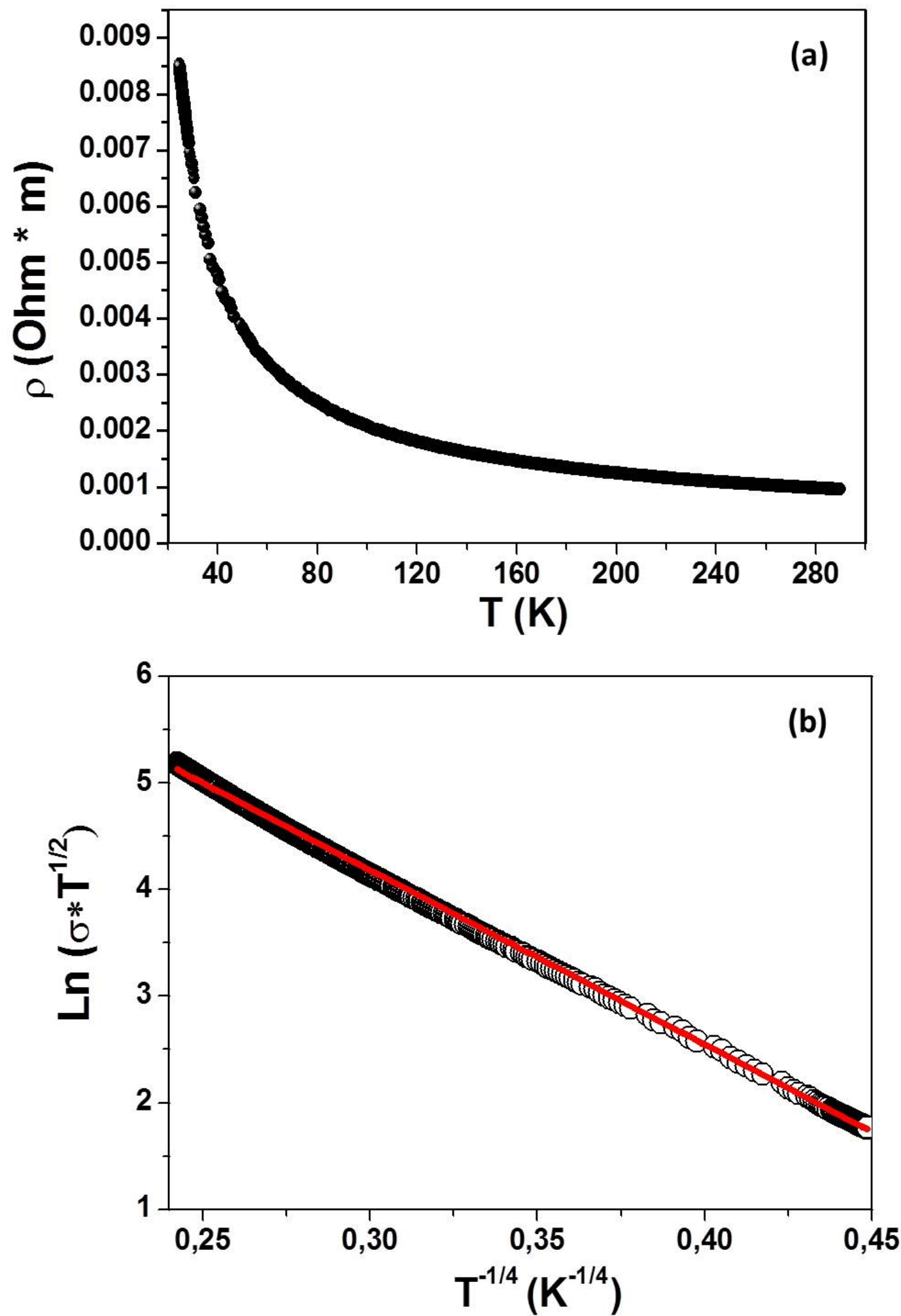

Figure 6. (a) Temperature variation of the resistivity of nanoplatelets with $5 \%$ oxygen content. (b) Fit (solid line) to the experimental data (open circles) using the Mott 3D VRH transport model in eq. (1). 


\section{Conclusions}

Oxidized-graphenic nanoplatelets have been synthesized using bamboo (Guadua angustifolia Kunth, macana biotype), which is a natural, cheap, highly renewable and abundant material. The HRTEM and EELS analyses have provided a complete structural and chemical description of these samples. We have shown that by varying the carbonization temperature, we can obtain OGNP with oxygen content below $20 \%$ and modify their local atomic structure. Nanoplatelets synthetized at the highest temperature of $973 \mathrm{~K}$ present lower oxygen content with short-range crystalline order and a carbon environment that is mainly in $\mathrm{sp}^{2}$ bonding configuration ( $\mathrm{sp}^{2}$ fraction of $87 \%$ ). On the other hand, nanoplatelets synthetized at the lowest temperature of $673 \mathrm{~K}$ present higher oxygen content and have a more disordered crystal structure with a mix of $\mathrm{sp}^{2}$ and $\mathrm{sp}^{3}$ characters ( $\mathrm{sp}^{2}$ fraction of 66\%). Moreover, we presented the electrical conductivity of single oxidized-graphenic nanoplatelets and investigated the effect of oxygen content via the combination of in-situ EDS measurements and electrical measurements. We found an increment by two orders of magnitude of the conductivity, reaching a value of $2.3 \times 10^{3} \mathrm{~S} / \mathrm{m}$ at the lowest oxygen atomic percentage of $5 \%$. The temperature dependence on conductivity showed typical semiconductor behavior, which could be described by the Mott three-dimensional variable-range hopping mechanism (3D-VRH). Estimations of bandgap energy showed variation from 0.30 to $0.11 \mathrm{eV}$ by decreasing oxygen content from 17 to $5 \%$. These results demonstrate that OGNP-BPA possess similar electrical behavior as conventionally obtained rGO sheets, which represents an significant advance towards commercially feasible and environmentally-sustainable fabrication of carbon nanomaterials from renewable natural source.

\section{Acknowledgements}

This work was supported by funds for internal calls for projects at Universidad del Valle IC10024, the Interdisciplinary Institute of Sciences at Universidad del Quindio and the Center of Excellence on Novel Materials at Universidad del Valle. The HRTEM and EELS analyses were conducted in the Laboratorio de Microscopías Avanzadas (LMA) at the Instituto de Nanociencia de Aragón (INA) - Universidad de Zaragoza (Spain). Some of the research leading to these results received funding from the European Union Seventh Framework Program under Grant Agreement 312483 - ESTEEM2 (Integrated Infrastructure Initiative - I3) and under Grant Agreement 604391 Graphene Flagship. R.A. also acknowledges funding from the Spanish Ministerio de Economia y Competitividad (FIS2013-46159C3-3-P), as well as from EU H2020 ETN project “Enabling Excellence” Grant Agreement 642742. Acknowledgment to Aragon regional government for funding the E26 group is given.

\section{References}

[1] Mao S, Pu H, and Chen J 2012 Graphene oxide and its reduction: modeling and experimental progress RSC Advances 2 2643-2662. 
[2] Singh V, Joung D, Zhai L, Das S, Khondaker S I and Seal S 2011 Graphene based materials: Past, present and future Progress in Materials Science 56 1178-1271.

[3] Gilje S, Han S, Wang M, Wang K L, Kaner R B 2007 A chemical route to graphene for device applications Nano Lett. 7 3394-3398.

[4] Gomez-Navarro C, Weitz R T, Bittner A M, Scolari M, Mews A, Burghard M, et al. 2007 Electronic transport properties of individual chemically reduced graphene oxide sheets Nano Lett. 7 3499-3503.

[5] Stankovich S, Dikin D A, Piner R D, Kohlhaas K A, Kleinhammes A, Jia Y, et al 2007 Synthesis of graphene-based nanosheets via chemical reduction of exfoliated graphite oxide Carbon 45 1558-1565.

[6] Stankovich S, Piner R D, Chen X Q, Wu N Q, Nguyen S T, Ruoff R S 2006 Stable aqueous dispersions of graphitic nanoplatelets via the reduction of exfoliated graphite oxide in the presence of poly(sodium 4-styrenesulfonate) J Mater Chem. 16 155-158.

[7] Eda G, Fanchini G and Chhowalla M 2008 Large-area ultrathin films of reduced graphene oxide as a transparent and flexible electronic material. Nature nanotechnol. 3 270-274.

[8] Becerril H A, Man J, Liu Z, Stoltenberg R M, Bao Z and Chen Y 2008 Evaluation of solutionprocessed reduced graphene oxide films as transparent conductors ACS Nano 2 463-470.

[9] Eda G and Chhowalla M 2010 Chemically derived Graphene Oxide: Towards large-Area Thin-Film Electronics and Optoelectronics. Adv. Mater. 22 2392-2415.

[10] He H, Klinowski J, Forster M \& Lerf A 1998 A new structural model for graphite oxide Chem. Phys. Lett. 287 53-56.

[11] Lerf A, He H, Forster M \& Klinowski J 1998 Structure of graphite oxide revisited J. Phys. Chem. B 102 4477-4482.

[12] Boukhvalov D W and Katsnelson M I 2008 Modeling of Graphite Oxide J. Am. Chem. Soc. 130 (32) 10697-10701.

[13] Yan J, Xian L, and Chou M Y 2009 Structural and electronic properties of oxidized graphene Phys. Rev. Lett. 103086802.

[14] Bagri A, Mattevi C, Acik M, Chabal Y J, Chowalla M and Shenoy V B 2010 Structural evolution during the reduction of chemically derived graphene oxide Nat. Chem. 2 581-587.

[15] Mattevi C, Eda G, Agnoli S, Miller S, Mkhoyan K A, Celik O, et al. 2009 Evolution of electrical, chemical, and structural properties of transparent and conducting chemically derived graphene thin films Adv. Funct. Matter. 19 2577-2583.

[16] Kaiser A B, Gomez-Navarro C, Sundaram R S, Burghard M, Kern K 2009 Electrical conduction mechanism in chemically derived graphene monolayers Nano Lett. 9 1787-1792. 
[17] Punckt C, Muckel F, Wolff S, Aksay I A, Chavarin C A, Bacher G, and Mertin W 2013 The effect of degree of reduction on the electrical properties of functionalized graphene sheets Appl. Phys. Lett. $102023114-5$.

[18] Jung I, Dikin D A, Piner R D and Ruoff R S 2008 Tunable Electrical Conductivity of Individual Graphene Oxide Sheets Reduced at “Low” Temperatures Nano Lett. 8 (12) 4283-4287.

[19] Park S \& Ruoff R S 2009 Chemical methods for the production of graphenes Nat. Nanotech. 4 217224.

[20] Kumar P V, Bardhan N M, Tongay S, Wu J, Belcher A M, Grossman J C 2014 Scalable enhancement of graphene oxide properties by thermally driven phase transformation Nat. Chem.6 (2) $151-8$.

[21] Kang H, Kulkarni A, Stankovich S, Ruoff R S, Baik S 2009 Restoring electrical conductivity of dielectrophoretically assembled graphite oxide sheets by thermal and chemical reduction techniques Carbon 47 1520-1525.

[22] Yan J-A and Chou M Y 2010 Oxidation functional groups on graphene: Structural and electronic properties Phys. Rev. B. 82125403.

[23] Hofmann U and Holst R 1939 The acid nature and methylation of graphitic oxide Ber. Dtsc. Chem. Ges. 72 754-771. doi:10. 1002/cber.19390720417

[24] Lerf A, He H, Riedl T, Forster M, Klinowski J N1997 MR studies of graphite oxide and its chemically modified derivatives Sol. Stat. Ion. 101(2) 857-862.

[25] Sholz W and Boehm H P 1969 Untersuchungen am graphitoxid betrachtungen zur struktur des graphitoxid Zeit. Anorg. Allg. Chem. 396(3) 327-340.

[26] Nakajima T and Matsuo N 1994 Formation process and structure of graphite oxide Carbon 32(3) 469-475.

[27] Ruess G 1946 Über das graphitoxyhydroxyd (graphitoxyd) Mntsf. Chem. Teil. Wssnchn. 76(3) 381417.

[28] T Szabo, Berkesi O, Forgo P, Josepovits K, Sanakis Y, Petridis D, Dekany I 2006 Evolution of surface functional groups in a series of progressively oxidized graphite oxides Chem. Mat. 18(11) 27402749.

[29] Navarro C G, Meyer J C, Sundaram R S, Chuvilin A, Kurasch S, Burghard M, Kern K, and Kaiser U 2010 Atomic structure of reduced graphene oxide Nano Lett. 10 1144-1148.

[30] Mkhoyan K A, Contryman A W, Silcox J, Stewart D A, Eda G, Mattevi C, Miller S, and Chhowalla M 2009 Atomic and Electronic Structure of Graphene-Oxide Nano Lett. 9 (3) 1058-1063.

[31] Wilson N R, Pandey P A, Beanland R, Young R J, Kinloch I A, Gong L, Liu Z, Suenaga K, Rourke J P, York S J, and Sloan J 2009 Graphene Oxide: Structural analysis and application as a highly transparent support for electron microscopy ACS Nano 3 (9) 2547-2556. 
[32] Xu Z, Bando Y, Liu L, Wang W, Bai X and Golberg D 2011 Electrical Conductivity, Chemistry, and Bonding Alternations under Graphene Oxide to Graphene Transition As Revealed by In Situ TEM ACS Nano 5 (6) 4401-4406.

[33] Erickson K, Erni R, Lee Z, Alem N, Gannett W and Zettl A 2010 Determination of the Local Chemical Structure of Graphene Oxide and Reduced Graphene Oxide Adv. Mater. 22 4467-4472.

[34] López V, Sundaram R S, Gómez-Navarro C, Olea D, Burghard M, Gómez-Herrero J, Zamora F and Kern K 2009 Chemical Vapor Deposition Repair of Graphene Oxide: A route to highly conductive graphene monolayers Adv. Mater. 21 4683-4686.

[35] Zhang Y, Wang D Q, Zhang S L, Wen Y H, Zhu Z Z 2014 Structures and electronic properties of oxidized graphene from first principles study Europhys. Lett. 105(3) 37005.

[36] Nourbakhsh A, Cantoro M, Vosch T, Pourtois G, Clemente F, van der Veen M H et al. 2010 Bandgap opening in oxygen plasma-treated graphene Nanotechnology 21435203.

[37] Huang H, Li Z, She J, and Wang W 2012 Oxygen density dependent band gap of reduced graphene oxide J. Appl. Phys. 111054317.

[38] Venugopala G, Krishnamoorthyb K, Kimb S-J 2013 An investigation on high-temperature electrical transport properties of graphene-oxide nano-thin films Applied Surface Science 280 903-908.

[39] Joung D and Khondaker S I 2012 Efros-Shklovskii variable-range hopping in reduced graphene oxide sheets of varying carbon sp2 fraction Phys. Rev. B. 86235423.

[40] C'iric' L, Sienkiewicz A, Gaa'l R, Jac'imovic' J, Va^ju C, Magrez A and Forro 2012 Defects and localization in chemically-derived graphene Phys. Rev. B. 86195139.

[41] Venugopala G, Krishnamoorthya K, Mohanc R, Kima S -J 2012 An investigation of the electrical transport properties of graphene-oxide thin films Materials Chemistry and Physics 132 29-33.

[42] Lopez V, Sundaram R S, Gómez-Navarro C, Olea D, Burghard M, Gómez-Herrero J, Zamora F, and Keedarn K 2009 Chemical Vapor Deposition Repair of Graphene Oxide: A Route to Highly Conductive Graphene Monolayers Adv. Mater. 21 4683-4686.

[43] Eda G, Mattevi C, Yamaguchi H, Kim H and Chhowalla M 2009 Insulator to semimetal transition in graphene oxide J. Phys. Chem. C 113 15768-15771.

[44] Mott N F 1968 Conduction in glasses containing transition metal ions J. Non-Cryst Solids 1 1-17.

[45] Efros A L and Shklovskii B I 1975 Coulomb gap and low temperature conductivity of disordered systems J. Phys. C: Solid State Phys. 8 L49-L51.

[46] Mott N F and Davis EA 1979 Electronic processes in non-crystalline materials, 2nd ed. (Clarendon Press: Oxford, UK). 
[47] Raj K G and Joy P A 2015 Cross over from 3D variable range hopping to the 2D weak localization conduction mechanism in disordered carbon with the extent of graphitization Phys. Chem. Chem. Phys. 17 16178-16185.

[48] Acik M and Chabal Y J A 2013 Review on Thermal Exfoliation of Graphene Oxide Journal of Materials Science Research 2 (1) 101-112.

[49] Prias-Barragan J J, Gross K, Ariza-Calderon H, and Prieto P 2016 Synthesis and vibrational response of graphite oxide platelets from bamboo for electronic applications Phys. Status Solidi A 213 (1) 85-90.

[50] Egerton R F 2011 Electron Energy-Loss Spectroscopy in the Electron Microscope (Springer Verlag)

[51] Lajaunie L, Pardanaud C, Martin C, Puech P, Hu C, Biggs MJ, and Arenal R Advanced Spectroscopic Analyses on a:C-H Materials: Revisiting the EELS Characterization and its Coupling with multi-wavelength Raman Spectroscopy, In Preparation.

[52] Fallon P and Walsh C A 1996 PEELS Program (University of Cambridge, England)

[53] Stephen AP 1980 PhD. Thesis (University of Cambridge, England)

[54] Egerton RF 2011 Electron Energy-Loss Spectroscopy in the Electron Microscope (Springer Verlag)

[55] Ferrari A, Libassi A, Tanner B, Stolojan V, Yuan J, Brown L, et al. 2000 Density, sp3 fraction, and cross-sectional structure of amorphous carbon films determined by $\mathrm{X}$-ray reflectivity and electron energy-loss spectroscopy Phys. Rev. B. 6211089.

[56] Titantah J, Lamoen D 2004 Determination of the electron effective band mass in amorphous carbon from density-functional theory calculations Phys. Rev. B. 70033101.

[57] Iakoubovskii K, Mitsuishi K, Nakayama Y, and Furuya K 2008 Thickness measurements with electron energy loss spectroscopy Microsc. Res. Tech. 71626.

[58] Lajaunie L, Boucher F, Dessapt R, and Moreau P 2013 Phys. Rev. B. 88115141.

[59] Hiely C I, Scanlon D O, Sokol A A, Woodley S M, Ganose A M, Ganose S, et al. 2015 Antiferromagnetism at $\mathrm{T}>500 \mathrm{~K}$ in the layered hexagonal ruthenate $\mathrm{SrRu}_{2} \mathrm{O}_{6}$ Phys. Rev. B. 92104413.

[60] Marcano N, Sangiao S, Plaza M, Pérez L, Fernández-Pacheco A, Córdoba R, et al. 2010 Weakantilocalization signatures in the magnetotransport properties of individual electrodeposited $\mathrm{Bi}$ Nanowires Appl. Phys. Lett. 96082110.

[61] De Teresa J M, Córdoba R, Fernández-Pacheco A, Montero O, Strichovanec P and Ibarra M R 2009 Origin of the Difference in the Resistivity of As-Grown Focused-Ion- and Focused-Electron-BeamInduced Pt Nanodeposits Journal of Nanomaterials 2009936863.

[62] Mayoral A and Arenal R 2015 Advanced Transmission Electron Microscopy: Applications to Nanomaterials (Eds. L. Francis, Springer).

[63] Arenal R, Blase X, and Loiseau A 2010 Boron-nitride and boron-carbonitride (BCN) NTs: synthesis, characterization and theory Advances in Physics 59101. 
[64] Wang Z, Dupré N, Lajaunie L, Moreau P, Martin JF, Boutafa L, Patoux S \& Guyomard D 2012 Effect of glutaric anhydride additive on the $\mathrm{LiNi}_{0.04} \mathrm{Mn}_{1.6} \mathrm{O}_{4}$ electrode/electrolyte interface evolution: A MAS NMR and TEM/EELS study J. Power Sources 215, 170-178.

[65] Panchakarla LS, Lajaunie L, Tenne R, and Arenal R 2015 Atomic Structural Studies on Thin Single-Crystalline Misfit-Layered Nanotubes of $\mathrm{TbS}^{-} \mathrm{CrS}_{2}$ The Journal of Physical Chemistry In Press, http://dx.doi.org/10.1021/acs.jpcc.5b05811.

[66] Liu A C Y, Arenal R, Miller D J, Chen X, Johnson J A, Erylmaz O L, Erdemir A, Woodford J B 2007 Structural order in near-frictionless DLC films probed at three different length scales in transmission electron microscope Phys. Rev. B. 75205402.

[67] Lajaunie L; Boucher F, Dessapt R, and Moreau P 2015 Quantitative Use of Electron Energy-Loss Spectroscopy Mo-M $\mathrm{M}_{2,3}$ Edges for the Study of Molybdenum Oxides Ultramicroscopy 149 1-8.

[68] Garvie L A, Craven A J, Brydson R 1994 Use of electron-energy loss near-edge fine structure in the study of minerals American Mineralogist. 79 411-25.

[69] Cody G, Ade H, Wirick S, Mitchell G, Davis A 1998 Determination of chemical-structural changes in vitrinite accompanying luminescence alteration using C-NEXAFS analysis Organic Geochemistry 28 441-55.

[70] Arenal R, De Matteis L, Custardoy L, Mayoral A, Tence M, Grazu V, De La Fuente J M, Marquina C, Ibarra M R 2013 Spatially-Resolved EELS Analysis of Antibody Distribution on Biofunctionalized Magnetic Nanoparticles ACS Nano 7 4006-4013.

[71] Robertson J 2002 Diamond-like amorphous carbon Mater. Sci. Eng. R. Rep. 37129.

[72] Ewels P, Sikora T, Serin V, Ewels C P and Lajaunie L A complete overhaul of the EELS and XAS database: eelsdb.eu. Microscopy and Microanalysis, In Press, http://dx.doi.org/10.1017/S1431927616000179

[73] Van Vechten A and Bergstresser T K 1970 Electronic Structures of Semiconductor Alloys Phys. Rev. B. 1(8) 3351-3358.

[74] Vurghaftman I, Meyer J R and Ram- Mohan L R J 2001 Appl. Phys. 89 5815-5875.

[75] Pandey D, Reifenberger R, and Piner R 2008 Scanning probe microscopy study of exfoliated oxidized graphene sheets Surf. Sci. 602 1607-1613. 